International Journal of Pure and Applied Mathematics

Volume 111 No. 2 2016, 189-197

ISSN: 1311-8080 (printed version); ISSN: 1314-3395 (on-line version)

url: http://www.ijpam.eu

doi: $10.12732 /$ ijpam.v111i2.5

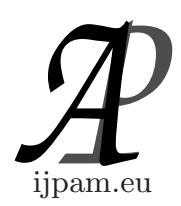

\title{
MAPPING PROPERTIES OF $\lambda$-SPIRALLIKE \\ AND $\lambda$-ROBERTSON FUNCTIONS OF \\ COMPLEX ORDER UNDER THE GENERALIZED \\ INTEGRAL OPERATOR
}

C. Selvaraj ${ }^{1}$, T.R.K. Kumar ${ }^{2} \S$

${ }^{1}$ Presidency College

Chennai, 600 005, INDIA

${ }^{2}$ R.M.K.Engineering College

R.S.M.Nagar, Kavaraipettai, 601206, INDIA

\begin{abstract}
In this paper, we define the some mapping properties of two subclasses of analytic function classes $\mathcal{S}_{\alpha}^{\lambda}(b)$ and $\mathcal{C}_{\alpha}^{\lambda}(b)$ under generalized integral operator.
\end{abstract}

AMS Subject Classification: $30 \mathrm{C} 45$

Key Words: analytic function, univalent function, bounded boundary rotation, generalized integral operator

\section{Introduction}

Let $\mathcal{A}$ denote the family of all functions of the form

$$
f(z)=z+\sum_{n=2}^{\infty} a_{n} z^{n} .
$$

which are analytic in the open unit disk $\mathbb{U}=\{z \in \mathbb{C}:|z|<1\}$ and satisfying the normalization conditions $f(0)=0$ and $f^{\prime}(0)=1$.

Received:

Revised:

July 8, 2016

October 16, 2016

Published: December 11, 2016

$\S$ Correspondence author (c) 2016 Academic Publications, Ltd. url: www.acadpubl.eu 
A function $f \in \mathcal{A}$ is said to be $\lambda$ - Spirallike function of complex order $\mathrm{b}$ and type $\alpha(0 \leq \alpha<1)$, denoted by $\mathcal{S}_{\alpha}^{\lambda}(b)$ (see [2]) if and only if

$$
\operatorname{Re}\left\{1+\frac{e^{i \lambda}}{b \cos \lambda}\left(\frac{z f^{\prime}(z)}{f(z)}-1\right)\right\}>\alpha \quad(z \in \mathbb{U}),
$$

for some real numbers $\lambda \in \mathbb{R}$ with $|\lambda|<\frac{\pi}{2}$ and $b \in \mathbb{C} \backslash\{0\}$.

A function $f \in \mathcal{A}$ is said to be $\lambda$ - Robertson functions of convex of complex order b and type $\alpha(0 \leq \alpha<1)$, denoted by $\mathcal{C}_{\alpha}^{\lambda}(b)$ (see [14]) if and only if

$$
\operatorname{Re}\left\{1+\frac{e^{i \lambda}}{b \cos \lambda}\left(\frac{z f^{\prime \prime}(z)}{f^{\prime}(z)}\right)\right\}>\alpha \quad(z \in \mathbb{U}),
$$

for some real numbers $\lambda \in \mathbb{R}$ with $|\lambda|<\frac{\pi}{2}$ and $b \in \mathbb{C} \backslash\{0\}$.

For $\mathrm{b}=1$ and $\lambda=0, S_{\lambda}^{*}(\rho, 1)=S^{*}(\rho)$ and $C_{\lambda}(\rho, 1)=C(\rho)$ the classes of functions that are starlike of order $\rho$ and convex of order $\rho$ in $\mathbb{U}$, respectively.

$$
C(\rho) \subset S^{*}(\rho) \quad(0 \leq \rho<1) .
$$

Notice that $S_{0}^{*}(b)=S^{*}(b)$ and $C_{0}(b)=C(b)$ the classes considered earliar by Nasr and Aouf [9] and Wiatrowski [15].

Let $P_{k}^{\lambda}(\rho)$ denote the class of functions $p: \mathbb{U} \longrightarrow \mathbb{C}$, analytic in $\mathbb{U}$ satisfying the following properties

1. $\mathrm{p}(0)=1$ and

2 .

$$
\int_{0}^{2 \pi}\left|\frac{\mathcal{R}\{p(z)-\rho\}}{1-\rho}\right| d \theta \leq k \pi, \quad z=r e^{i \theta} \in \mathbb{U}
$$

where $k \geq 2,0 \leq \rho<1, \lambda$ is real with $|\lambda|<\frac{\pi}{2}$. For $\lambda=0$, this class was introduced in [17] and for $\rho=0$, see [13]. For $k=2, \lambda=0$ and $\rho=0$, the class $P_{k}^{\lambda}(\rho)$ reduces to the class $\mathrm{P}$ of functions with positive real part and the class $k=2$ gives us the class $P(\rho)$ of functions with positive real part greater than $\rho$. For $k>2$, the functions in $P_{k}$ may not have positive real part.

Definition 1. A function $f(z) \in \mathcal{A}$ is said to be in the class $\mathcal{S}_{\alpha}^{\lambda}(b)$, $(b \in \mathbb{C} \backslash\{0\})$ if and only if

$$
1+\frac{e^{i \lambda}}{b \cos \lambda}\left(\frac{z f^{\prime}(z)}{f(z)}-1\right) \in P_{k}^{\lambda}(\rho) \quad(k>2, \quad 0 \leq \rho<1) .
$$

Notice that $\mathcal{S}_{\alpha}^{0}(1)=\mathcal{S}_{\alpha}^{*}$, which is the class of functions with bounded radius rotation. 
Definition 2. A function $f(z) \in \mathcal{A}$ is said to be in the class $\mathcal{C}_{\alpha}^{\lambda}(b)$ $(b \in \mathbb{C} \backslash\{0\})$ if and only if

$$
1+\frac{e^{i \lambda}}{b \cos \lambda}\left(\frac{z f^{\prime \prime}(z)}{f^{\prime}(z)}\right) \in P_{k}^{\lambda}(\rho) \quad(k>2, \quad 0 \leq \rho<1) .
$$

Notice that $\mathcal{C}_{\alpha}^{0}(1)=\mathcal{C}_{\alpha}$, which is the class of functions with bounded radius rotation.

First discussed by Paatero [3]. It is clear that

$$
f(z) \in \mathcal{C}_{\alpha}^{\lambda}(b) \Longleftrightarrow z f^{\prime}(z) \in \mathcal{S}_{\alpha}^{\lambda}(b) .
$$

Recently, Frasin[8] introduced the following generalized integral operators:

Let $\alpha_{i}, \beta_{i} \in \mathbb{C}$ for all $i=1,2,3, \cdots, n, n \in \mathbb{N}, \gamma \in \mathbb{C}$ with $\mathcal{R}(\gamma)>0$.

Let $\mathcal{I}_{\gamma}^{\alpha_{i}, \beta_{i}}: \mathcal{A}^{n} \longrightarrow \mathcal{A}$ be the integral operator defined by

$$
\begin{gathered}
\mathcal{I}_{\gamma}^{\alpha_{i}, \beta_{i}}\left(f_{1}, f_{2}, \cdots, f_{n}\right)(z) \\
=\left\{\int_{0}^{z} \gamma t^{\gamma-1}\left(f_{1}^{\prime}(t)\right)^{\alpha_{1}}\left(\frac{f_{1}(t)}{t}\right)^{\beta_{1}} \cdots\left(f_{n}^{\prime}(t)\right)^{\alpha_{n}}\left(\frac{f_{n}(t)}{t}\right)^{\beta_{n}} d t\right\}^{\frac{1}{\gamma}},
\end{gathered}
$$

where the power is taken as the principal one.

Notice that, the integral operator $\mathcal{I}_{\gamma}^{\alpha_{i}, \beta_{i}}\left(f_{1}, f_{2}, \cdots, f_{n}\right)(z)$ generalizes several previously studied operators as follows:

1. For $\alpha_{i}=0$ for all $i=1,2,3, \ldots n$, the integral operator

$$
\mathcal{I}_{\gamma}^{0, \beta_{i}}\left(f_{1}, f_{2}, \cdots, f_{n}\right)(z)=\mathcal{I}_{\gamma}\left(f_{1}, f_{2}, \cdots, f_{n}\right)(z),
$$

where

$$
\begin{aligned}
& \mathcal{I}_{\gamma}\left(f_{1}, f_{2}, \cdots, f_{n}\right)(z) \\
& =\left\{\int_{0}^{z} \gamma t^{\gamma-1}\left(\frac{f_{1}(t)}{t}\right)^{\beta_{1}} \cdots\left(\frac{f_{n}(t)}{t}\right)^{\beta_{n}} d t\right\}^{\frac{1}{\gamma}},
\end{aligned}
$$

is introduced and studied by Breaz and Breaz [4].

2. For $\alpha_{i}=0$ for all $i=1,2,3, \ldots n$ and $\gamma=1$, the integral operator $\mathcal{I}_{1}^{0, \beta_{i}}\left(f_{1}, f_{2}, \cdots, f_{n}\right)(z)=\mathcal{F}_{n}(z)$, where

$$
\mathcal{F}_{n}(z)=\int_{0}^{z}\left(\frac{f_{1}(t)}{t}\right)^{\beta_{1}} \cdots\left(\frac{f_{n}(t)}{t}\right)^{\beta_{n}} d t
$$

is introduced and studied by Breaz and Breaz [4]. 
3. For $\beta_{i}=0$ for all $i=1,2,3, \ldots n$ and $\gamma=1$, the integral operator $\mathcal{I}_{1}^{\alpha_{i}, 0}\left(f_{1}, f_{2}, \cdots, f_{n}\right)(z)=\mathcal{F}_{\alpha_{1}, \alpha_{2}, \cdots \alpha_{n}}(z)$, where

$$
\mathcal{F}_{\alpha_{1}, \alpha_{2}, \cdots \alpha_{n}}(z)=\int_{0}^{z}\left(f_{1}^{\prime}(t)\right)^{\alpha_{1}} \cdots\left(f_{n}^{\prime}(t)\right)^{\alpha_{n}} d t
$$

is introduced and studied by Breaz and Breaz et al [6].

Recently, Breaz and Güney [5] considered the integral operators $\mathcal{F}_{n}(z)$ and $\mathcal{F}_{\alpha_{1}, \alpha_{2}, \cdots \alpha_{n}}(z)$ and obtained their properties on the classes $\mathcal{S}_{\delta}^{*}(b)$ and $\mathcal{C}_{\delta}(b)$ of starlike and convex functions of complex order b and type $\delta$. Later on Noor et al. [11] considered the same integral operators and investigated the mapping properties for the classes $\mathcal{V}_{k}^{\lambda}(\rho, b)$ and $\mathcal{R}_{k}^{\lambda}(\rho, b)$.

Motivated by the aforementioned work, in this paper, the author investigates some mapping properties of the classes $\mathcal{V}_{k}^{\lambda}(\rho, b)$ and $\mathcal{R}_{k}^{\lambda}(\rho, b)$ under generalized integral operator defined in (8) when $\gamma=1$. The results obtained in this paper are generalized results of Breaz and Güney [5] and Noor et al. [11].

We recall the following lemma which is useful for our investigation:

Lemma 3. ([12]). Let $f(z) \in \mathcal{V}_{k}(\alpha), 0 \leq \alpha<1$. Then $f(z) \in \mathcal{R}_{k}(\beta)$ where

$$
\beta=\frac{1}{4}\left[(2 \alpha-1)+\sqrt{4 \alpha^{2}-4 \alpha+9}\right] .
$$

\section{Main Results}

Theorem 4. Let $f_{i}, \phi_{i} \in \mathcal{S}_{\alpha}^{\lambda}(b)$ for all $i=1,2,3, \ldots n$ with $0 \leq \rho<1$, $b \in \mathbb{C} \backslash\{0\}$ and $\alpha_{i}, \beta_{i} \in \mathbb{R}^{+}$for $1 \leq i \leq n$. If

$$
0 \leq(\rho-1) n+(\rho-1) \sum_{i=1}^{n} \beta_{i}+1<1
$$

then the integral operator

$$
\mathcal{I}_{1}^{\alpha_{i}, \beta_{i}}\left(f_{1}, f_{2}, \cdots, f_{n}\right)(z)=\int_{0}^{z} \prod_{i=1}^{n}\left(f_{i}^{\prime}(t)\right)^{\alpha_{i}}\left(\frac{f_{i}(t)}{t}\right)^{\beta_{i}} d t
$$

belong to the class $\mathcal{C}_{\psi}^{\lambda}(b)$ with

$$
\psi=1+(\rho-1) n+(\rho-1) \sum_{i=1}^{n} \beta_{i} .
$$


Proof. For the sake of simplicity, in the proof, we shall write $\mathcal{H}(z)$ instead of $\mathcal{I}_{1}^{\alpha_{i}, \beta_{i}}\left(f_{1}, f_{2}, \cdots, f_{n}\right)(z)$.

Diffirentiation (13) with respect to z, we obtain

$$
\mathcal{H}^{\prime}(z)=\prod_{i=1}^{n}\left(f_{i}^{\prime}(z)\right)^{\alpha_{i}}\left(\frac{f_{i}(z)}{z}\right)^{\beta_{i}} .
$$

Let us define

$$
\phi_{i}(z)=z\left(f_{i}^{\prime}(z)\right)^{\alpha_{i}} .
$$

Clearly, $\phi_{i}(z) \in \mathcal{A}$.Equation (2.4) becomes

$$
\mathcal{H}^{\prime}(z)=\prod_{i=1}^{n} \frac{\phi_{i}(z)}{z^{n}}\left(\frac{f_{i}(z)}{z}\right)^{\beta_{i}} .
$$

Logarithmic differentiation of (16) yields

$$
\frac{\mathcal{H}^{\prime \prime}(z)}{\mathcal{H}^{\prime}(z)}=\sum_{i=1}^{n}\left[\beta_{i}\left(\frac{f_{i}^{\prime}(z)}{f_{i}(z)}-\frac{1}{z}\right)+\left(\frac{\phi_{i}^{\prime}(z)}{\phi_{i}(z)}-\frac{1}{z}\right)\right] .
$$

Multiplying (17) by $z$ and simplifying, we get

$$
\begin{aligned}
& 1+\frac{e^{i \lambda}}{b \cos \lambda}\left(\frac{z \mathcal{H}^{\prime \prime}(z)}{\mathcal{H}^{\prime}(z)}\right)=1-n-\sum_{i=1}^{n} \beta_{i} \\
& +\sum_{i=1}^{n}\left\{\beta_{i}\left[1+\frac{e^{i \lambda}}{b \cos \lambda}\left(\frac{z f_{i}^{\prime}(z)}{f_{i}(z)}-1\right)\right]+1+\frac{e^{i \lambda}}{b \cos \lambda}\left(\frac{z \phi_{i}^{\prime}(z)}{\phi_{i}(z)}-1\right)\right\} .
\end{aligned}
$$

Adding and subtracting $\alpha$ on the right hand side of (18) gives

$$
\begin{aligned}
& {\left[\left(1+\frac{e^{i \lambda}}{b \cos \lambda}\left(\frac{z \mathcal{H}^{\prime \prime}(z)}{\mathcal{H}^{\prime}(z)}\right)\right)-\psi\right]} \\
& =\sum_{i=1}^{n} \beta_{i}\left[\left(1+\frac{e^{i \lambda}}{b \cos \lambda}\left(\frac{z f_{i}^{\prime}(z)}{f_{i}(z)}-1\right)\right)-\alpha\right] \\
& +\sum_{i=1}^{n}\left[\left(1+\frac{e^{i \lambda}}{b \cos \lambda}\left(\frac{z \phi_{i}^{\prime}(z)}{\phi_{i}(z)}-1\right)-\alpha\right)\right]
\end{aligned}
$$

where $\psi$ is given by (14). Taking real part of (20) and after simplification, we get

$$
\int_{0}^{2 \pi}\left|\operatorname{Re}\left[\left(1+\frac{e^{i \lambda}}{b \cos \lambda}\left(\frac{z \mathcal{H}^{\prime \prime}(z)}{\mathcal{H}^{\prime}(z)}\right)\right)-\psi\right]\right| d \theta
$$




$$
\begin{aligned}
& \leq \sum_{i=1}^{n} \beta_{i} \int_{0}^{2 \pi}\left|\operatorname{Re}\left[\left(1+\frac{e^{i \lambda}}{b \cos \lambda}\left(\frac{z f_{i}^{\prime}(z)}{f_{i}(z)}-1\right)\right)-\alpha\right]\right| d \theta \\
& \quad+\sum_{i=1}^{n} \int_{0}^{2 \pi}\left|\operatorname{Re}\left[\left(1+\frac{e^{i \lambda}}{b \cos \lambda}\left(\frac{z \phi_{i}^{\prime}(z)}{\phi_{i}(z)}-1\right)\right)-\alpha\right]\right| d \theta .
\end{aligned}
$$

Since, by hypothesis $f_{i}, \phi_{i} \in \mathcal{S}_{\alpha}^{\lambda}(b)$ for $1 \leq i \leq n$, we have

$$
\begin{aligned}
& \int_{0}^{2 \pi}\left|\operatorname{Re}\left[\left(1+\frac{e^{i \lambda}}{b \cos \lambda}\left(\frac{z f_{i}^{\prime}(z)}{f_{i}(z)}-1\right)\right)-\alpha\right]\right| d \theta \leq(1-\alpha) k \pi, \\
& \int_{0}^{2 \pi}\left|\operatorname{Re}\left[\left(1+\frac{e^{i \lambda}}{b \cos \lambda}\left(\frac{z \phi_{i}^{\prime}(z)}{\phi_{i}(z)}-1\right)\right)-\alpha\right]\right| d \theta \leq(1-\alpha) k \pi .
\end{aligned}
$$

Using (22) and (23) in (21), we have

$$
\int_{0}^{2 \pi}\left|\mathcal{R}\left[\left(1+\frac{e^{i \lambda}}{b \cos \lambda}\left(\frac{z \mathcal{H}^{\prime \prime}(z)}{\mathcal{H}^{\prime}(z)}\right)\right)-\psi\right]\right| d \theta \leq(1-\psi) k \pi,
$$

where $\psi$ is given by (14). Hence $\mathcal{H} \in \mathcal{S}_{\alpha}^{\lambda}(b)$. Thus, the proof of Theorem 4 is completed.

Put $\alpha_{i}=0$ for all $i=1,2,3, \ldots n$ in Theorem 2.1. Notice that, in such case $\mathcal{H}(z)=\mathcal{F}_{n}(z)$ and $\phi_{i}(z)=z$ which shows $\frac{z \phi_{i}(z)}{\phi_{i}(z)}-1=0$.

Therefore, from (20), it follows that

$$
\begin{aligned}
& {\left[\left(1+\frac{e^{i \lambda}}{b \cos \lambda}\left(\frac{z \mathcal{H}^{\prime \prime}(z)}{\mathcal{H}^{\prime}(z)}\right)\right)-\psi\right]} \\
& =\sum_{i=1}^{n} \beta_{i}\left[\left(1+\frac{e^{i \lambda}}{b \cos \lambda}\left(\frac{z f_{i}^{\prime}(z)}{f_{i}(z)}-1\right)\right)-\alpha\right],
\end{aligned}
$$

where $\psi=1+(1-\alpha) \sum_{i=1}^{n} \beta_{i}$.

Corollary 5. (cf. [11], Theorem 2.1). Let $f_{i}(z) \in \mathcal{S}_{\alpha}(b)$ for all $1 \leq i<n$ with $0 \leq \rho<1, b \in \mathbb{C} \backslash\{0\}$ and $\beta_{i}>0,1 \leq i<n$. If

$$
0 \leq 1+(\rho-1) \sum_{i=1}^{n} \beta_{i}<1
$$

then $\mathcal{F}_{n}(z) \in \mathcal{C}_{\delta}^{\lambda}(b)$ with $\delta=1+(1-\rho) \sum_{i=1}^{n} \beta_{i}$. 
Next, we take $\beta_{i}=0,1 \leq i \leq n$ in Theorem 4. In this case

$$
\mathcal{H}(z)=\mathcal{F}_{\alpha_{1}, \alpha_{2}, \cdots \alpha_{n}}(z)=\int_{0}^{z} \prod_{i=1}^{n}\left(f_{i}^{\prime}(z)\right)^{\alpha_{i}} d z
$$

which implies $\mathcal{H}^{\prime}(z)=\prod_{i=1}^{n} \frac{\phi_{i}(z)}{z^{n}}$.

$$
\frac{z \mathcal{H}^{\prime \prime}(z)}{\mathcal{H}^{\prime}}=\sum_{i=1}^{n}\left(\frac{z \phi_{i}^{\prime}(z)}{\phi_{i}(z)}-1\right)=\sum_{i=1}^{n} \alpha_{i} \frac{z f_{i}^{\prime \prime}(z)}{f_{i}^{\prime}(z)}
$$

we have the following results due to Noor et al. [11].

Corollary 6. (cf. [11], Theorem 2.5). Let $f_{i}(z) \in \mathcal{C}_{\alpha}(b)$ for all $1 \leq i<n$ with $0 \leq \rho<1, b \in \mathbb{C} \backslash\{0\}$ and $\alpha_{i}>0,1 \leq i<n$. If

$$
0 \leq 1+(\rho-1) \sum_{i=1}^{n} \alpha_{i}<1
$$

then $\mathcal{F}_{\alpha_{1}, \alpha_{2}, \cdots \alpha_{n}}(z) \in \mathcal{C}_{\delta_{1}}(b)$ with $\delta_{1}=1+(1-\rho) \sum_{i=1}^{n} \alpha_{i}$.

Remark 7. If $k=2$, in Corollary 2.2, we obtain the results of ([5], Theorem 1) and ([11], Corollary 2.2).

Remark 8. If $k=2$, in Corollary 2.3, we obtain the results of ([5], Theorem 3) and ([11], Corollary 2.6).

Theorem 9. Let $f_{i}, \phi_{i} \in \mathcal{C}_{\alpha}(1)$ for all $i=1,2,3, \ldots n$ with $0 \leq \alpha<1$. Let $\alpha_{i}, \beta_{i} \in R e^{+}$for $1 \leq i \leq n$. If

$$
0 \leq(\beta-1) \sum_{i=1}^{n}\left(1+\beta_{i}\right)+1<1
$$

then, $\mathcal{H}(z) \in \mathcal{C}_{l}(1)$ with $l=1+(\beta-1)+\sum_{i=1}^{n}\left(1+\beta_{i}\right)$ and $\beta$ is given by $(11)$.

Proof. proceeding as Theorem 4 with $b=1$ and $\lambda=0$. we have

$$
\begin{aligned}
& \int_{0}^{2 \pi}\left|\operatorname{Re}\left[\left(1+\left(\frac{z \mathcal{H}^{\prime \prime}(z)}{\mathcal{H}^{\prime}(z)}\right)\right)-l\right]\right| d \theta \\
& \leq \sum_{i=1}^{n} \beta_{i} \int_{0}^{2 \pi}\left|\operatorname{Re}\left[\left(\frac{z f_{i}^{\prime}(z)}{f_{i}(z)}\right)-\beta\right]\right| d \theta \\
& \quad+\sum_{i=1}^{n} \int_{0}^{2 \pi}\left|\operatorname{Re}\left[\left(\frac{z \phi_{i}^{\prime}(z)}{\phi_{i}(z)}\right)-\beta\right]\right| d \theta .
\end{aligned}
$$


Since, by hypothesis $f_{i}, \phi_{i} \in \mathcal{C}_{\alpha}(l)$ for $1 \leq i \leq n$ and by using Lemma 3 , we have

$$
\int_{0}^{2 \pi}\left|\operatorname{Re}\left[\left(\frac{z f_{i}^{\prime}(z)}{f_{i}(z)}\right)-\beta\right]\right| d \theta \leq(1-\beta) k \pi,
$$

and

$$
\int_{0}^{2 \pi}\left|\operatorname{Re}\left[\left(\frac{z \phi_{i}^{\prime}(z)}{\phi_{i}(z)}\right)-\beta\right]\right| d \theta \leq(1-\beta) k \pi,
$$

and $\beta$ is given by (11). Using (27) and (28) in (26), we obtain

$$
\int_{0}^{2 \pi}\left|\operatorname{Re}\left[\left(1+\left(\frac{z \mathcal{H}^{\prime \prime}(z)}{\mathcal{H}^{\prime}(z)}\right)\right)-l\right]\right| d \theta \leq(1-l) k \pi .
$$

Thus, $\mathcal{H}(z) \in \mathcal{C}^{\lambda}(1, l)$ with $l=1+(\beta-1)+\sum_{i=1}^{n}\left(1+\beta_{i}\right)$.

Remark 10. For $\alpha_{i}=0$, we obtain the results of ([11], Theorem 2.3).

For $n=1, \lambda=0, \alpha_{1}=0, \beta_{1}=0, f_{1}=f$ in Theorem 9 . We get the following results due to $[11]$.

Corollary 11. [11]. Let $f(z) \in \mathcal{C}_{\alpha}(1)$. Then the Alexander operator $\mathcal{I}(z)=\int_{0}^{z} \frac{f(t)}{t} d t$ (see [1]) belongs to the class $\mathcal{C}_{\alpha}(1)$, where $\beta$ is given by (11).

Remark 12. For $\alpha=0$ and $k=2$, in the above Corollary 11, we have the well known result $f(z) \in \mathcal{C}(0) \Rightarrow \mathcal{I}(z) \in \mathcal{C}\left(\frac{1}{2}\right)$.

\section{References}

[1] W. Alexander, Function which map the interior of the unit circle upon simple regions, Ann. Math., 17(1915), 12-22.

[2] F. M. Al-Oboudi and M. M. Haidan, Spirallike functions of complex order, Journal of Natural Geometry, 19(2001), no. 1-2, 53-72.

[3] S. D. Bernardi, New distortion theorem for functions of positive real part and applications to the partial sums of univalent convex functions, Amer. Math. Soc., 45 (1974), 113-118.

[4] D. Breaz and N. Breaz, Two integral operators, Studia Universitatis Babes-Bolyai. Mathematica, Cluj-Napoca, 3 (2002), 13-21.

[5] D. Breaz and H. Ö. Güney, The integral operator on the classes $\mathcal{S}_{\alpha}^{*}(b)$ and $\mathcal{C}_{\alpha}(b)$, J.Math. Ineq., 2 (2008), 97-100.

[6] D. Breaz, S. Owa and N. Breaz, A new integral univalent operator, Acta Univ. Apul., 16 (2008), 11-16.

[7] B. A. Frasin, Family of analytic functions of complex order, Acta Math. Acad. Paedagog.Nyházi. (N.S.) 22 (2006), no. 2, 179-191. 
[8] B. A. Frasin, Order of convexity and univalency of general integral operator, J. Franklin Inst. 348 (2011), 1013-1019.

[9] M. Nasr and M. Aouf, Starlike function of complex order. J. Nat. Sci. Math., 25 (1985), $1-12$.

[10] K. I. Noor, On subclasses of close-to-convex functions of higher order, Int. J. Math. Sci. 15, (1992), 279-290.

[11] K. I. Noor, M. Arif and W. Ul. Haq, Some properties of certain integral operators, Acta Univ. Apul. 21, (2010), 89-95.

[12] K. I. Noor, W. Ul. Haq, M. Arif and S. Mustafa, On bounded boundary and bounded radius rotations, J. Inequal. Appl. 2009, Art.ID 813687, 12 pp.

[13] B.Pinchuk, Functions with bounded boundary rotation, Israel. J. Math., 10, (1971), $7-16$.

[14] M. S. Robertson, Univalent functions $f(z)$ for which $z f^{\prime}(z)$ is spirallike, The Michigan Math. Journal 16, (1969), 97-101.

[15] P. Wiatrowski, On the coefficients of some family of holomorphic functions, Zeszyty Nauk. Uniw. Lodz-Nauk. Mat.-przyrod, 2, (1970), 75-85.

[16] T.Panigrahi, G.Murugusundaramoorthy, Mapping properties of the general integral operator on the classes $\mathcal{R}_{k}(\rho, b)$ and $\mathcal{V}_{k}(\rho, b)$, Applied Mathematics E-Notes, 15, (2015), $14-21$.

[17] K. Padmanabhan and R. Parvatham, Properties of a class of functions with bounded boundary rotation, Ann. Polon. Math., 31,(1975), 311-323. 
